\title{
Efectos de la inclusión de ejercicios de salto con y sin sobrecarga externa en el calentamiento sobre parámetros de rendimiento físico en atletas jóvenes de balonmano
}

\author{
Gómez-Álvarez, Nicolás; Moyano, Francisco; Huichaqueo, Elías; Veruggio, Matías; Urrutia, Víctor; \\ Hermosilla-Palma, Felipe; Pavez-Adasme, Gustavo \\ Efectos de la inclusión de ejercicios de salto con y sin sobrecarga externa en el calentamiento sobre parámetros \\ de rendimiento físico en atletas jóvenes de balonmano \\ MHSalud, vol. 17, núm. 1, 2020 \\ Universidad Nacional, Costa Rica \\ Disponible en: http://www.redalyc.org/articulo.oa?id=237061117004 \\ DOI: https://doi.org/10.15359/mhs.17-1.4
}

Esta obra está bajo una Licencia Creative Commons Atribución-NoComercial-SinDerivar 3.0 Internacional. 
MHSALUD, ISSN: 1659-097X, 17(1), ENERo-Junio, 2020, PP 1-12 Gómez-Álvarez, Moyano, Huichaqueo,Veruggio, Urrutia, Hermosilla-Palma, Pavez-Adasme

\section{Efectos de la inclusión de ejercicios de salto con y sin sobrecarga externa en el calentamiento sobre parámetros de rendimiento físico en atletas jóvenes de balonmano}

Effects of The Inclusion of Jump Exercises with and without External Overload in The Warm-Up on Physical Performance Parameters in Young Handball Athletes

Efeitos da inclusão de exercícios de salto com e sem pesos externo no aquecimento sobre os parâmetros de desempenho físico de jovens atletas de handebol

Nicolás Gómez-Álvarez

Universidad Adventista de Chile, Chile

nicolasgomez@unach.cl

(iD) http://orcid.org/0000-0003-3987-4189

Francisco Moyano

Universidad Adventista de Chile, Chile

franciscomoyano@alu.unach.cl

(D) http://orcid.org/0000-0002-8303-7911

Elías Huichaqueo

Universidad Adventista de Chile, Chile

eliashuichaqueo@alu.unach.cl

(iD http://orcid.org/0000-0003-3022-6986

Matias Veruggio

Universidad Adventista de Chile, Chile

matiasveruggio@alu.unach.cl

(iD) http://orcid.org/0000-0002-5186-4998

Victor Urrutia

Universidad Adventista de Chile, Chile

victorurrutia@alu.unach.cl

(iD http://orcid.org/0000-0002-8739-4879

Felipe Hermosilla-Palma

Universidad Pedro de Valdivia, Chile

fhermosilla@upv.cl

(DD http://orcid.org/0000-0001-8686-6793

Gustavo Pavez-Adasme

Universidad Adventista de Chile, Chile

gustavopavez@unach.cl

(iD http://orcid.org/0000-0003-4377-384X
DOI: https://doi.org/10.15359/mhs.17-1.4

Redalyc: http://www.redalyc.org/articulo.oa? id $=237061117004$
Recepción: 28 Junio 2019

Aprobación: 31 Octubre 2019

\section{RESUMEN:}

El objetivo de este estudio fue comparar los efectos de un calentamiento con aplicación de saltos cargados y sin carga sobre el tiempo en sprint de 20 metros, saltos horizontales y saltos verticales en jugadores juveniles de balonmano. La muestra estaba conformada 
MHSALUD, ISSN: 1659-097X, 17(1), ENERO-JUNIO, 2020, PP 1-12

Gómez-Álvarez, Moyano, Huichaqueo,Veruggio, Urrutia, Hermosilla-Palma, Pavez-Adasme

por 13 jugadores de balonmano (16,2 \pm 1,3 años) de San Carlos. Se realizó un estudio experimental con un diseño crossover aleatorizado. Se aplicaron 3 protocolos de calentamiento distintos, que implicaban correr más estiramientos dinámicos (CE); correr, estiramientos dinámicos y saltos (CES), y, finalmente, correr, estiramientos dinámicos y saltos cargados con el $8 \%$ de su peso corporal (CESH). Para las evaluaciones pre- y poscalentamiento, se utilizó un test de salto contramovimiento (CMJ), salto horizontal (SH) y sprint de 20 metros cronometrado. Los resultados muestran diferencias significativas entre pre- y postest para los 3 grupos en el SH (diferencias para CES: 0,09 \pm 0,12; CESH: 0,12 \pm 0,16 y CE: 0,07 $\pm 0,16$ ), mientras que solo CES y CESH mejoraron significativamente la altura en CMJ y tiempo en el sprint de 20 metros (diferencia para CMJ: 2,95 $\pm 0,56$ y 3,50 $\pm 0,73$; y sprint de $20 \mathrm{~m}: 0,29 \pm 0,31$ y 0,20 $\pm 0,26$, respectivamente). En conclusión, un calentamiento que incluye distintos tipos de saltos es eficaz para mejorar $\mathrm{SH}, \mathrm{CMJ}$ y velocidad en 20 metros. Es necesario realizar más investigación, para determinar beneficios específicos de saltos con halteras de manos que mejoren el rendimiento físico.

Palabras Clave: calentamiento, ejercicio físico, atletas, rendimiento.

\section{Abstract:}

The aim of this study is to compare the effects of a warm-up with the application of loaded and unloaded jumps on the performance in speed, horizontal jumps and vertical jumps in youth handball players. The sample consists of 13 handball players from the city of San Carlos (16.2 \pm 1.3 years). An experimental study with a randomized crossover design carried out. 3 different heating protocols applied, which involved running more dynamic stretching (RS), running, dynamic stretching and jumping (RSJ) and finally running, dynamic stretching and jumps loaded with $8 \%$ of your body weight (RSLJ). For the pre- and post-warming assessments, a countermovement jump test $(\mathrm{CMJ})$, horizontal jump $(\mathrm{HJ})$ and were used through a 20-meter timed to sprint test. The results show significant differences between pre- and post-test for the 3 groups in the SH (difference for CES: $0.09 \pm 0.12$; CESH: 0.12 \pm 0.16 and EC: $0.07 \pm 0.16$ ), while only CES and CESH significantly improved the height in CMJ and sprint time of 20 meters (difference for CMJ: $2.95 \pm 0.56$ and $3.50 \pm 0.73$; and sprint 20 meters: $0.29 \pm 0,31$ and $0.20 \pm 0.26$, respectively). In conclusion, a warm-up that includes different types of jumps is effective in improving SH, CMJ and speed in 20 meters. More research needed to determine specific benefits of handheld loading jumps to improve physical performance.

KEYWORDS: warm-up, physical exercise, athletes, performance.

\section{Resumo:}

O objetivo deste estudo foi comparar os efeitos de um aquecimento com a aplicação de saltos acompanhado e não acompanhados de pesos, em velocidade de 20 metros de tempo, saltos horizontais e saltos verticais em jogadores de handebol juvenil. A amostra

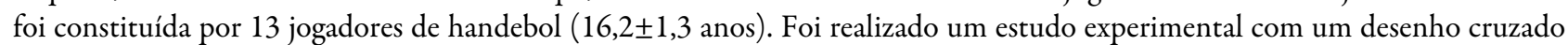
randomizado. Foram aplicados três protocolos diferentes de aquecimento, envolvendo alongamentos mais dinâmicos (CE), alongamentos e saltos dinâmicos (CES) e, finalmente, alongamentos e saltos dinâmicos (CESH), carregados com $8 \%$ do seu peso corporal. Para as avaliações antes e depois do aquecimento, foram utilizados um salto contra movimento (CMJ), salto horizontal (SH) e um sprint temporizado de 20 metros. Os resultados mostram diferenças significativas entre o pré e após a prova para

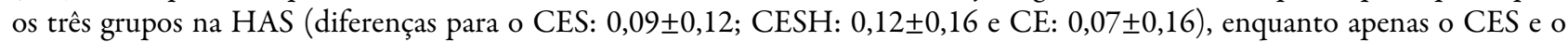
CESH melhoraram significativamente a altura na CMJ e o tempo de velocidade de 20 metros (diferença para CMJ: 2,95 $\pm 0,56$

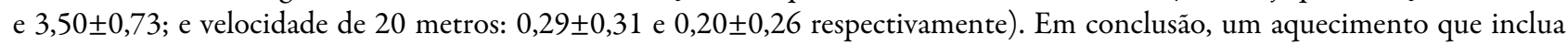
diferentes tipos de saltos é eficaz na melhoria do SH, CMJ e velocidade em 20 metros. Mais pesquisas são necessárias para determinar os benefícios específicos de saltar com pesos para melhorar o desempenho físico.

Palavras-chave: Aquecimento, exercício físico, atletas, rendimento.

\section{INTRODUCCIÓN}

Los deportes de equipo y carácter intermitente como el balonmano están caracterizados por movimientos de alta intensidad que incluyen carreras de velocidad con periodos de aceleración y desaceleración, saltos y cambios de dirección, respondiendo a un juego complejo y multifactorial (Wagner, Finkenzeller, Würth, \& Von Duvillard, 2014). Estas características deportivas requieren que los jugadores desarrollen una adecuada preparación previa, para obtener los mejores resultados deportivos desde el principio de la competición. El calentamiento es una estrategia habitualmente utilizada, con el fin de generar efectos agudos positivos en el rendimiento de los jugadores (Pagaduan, Pojskić, Užičanin Babajić, 2012), el que, habitualmente, está caracterizado por un conjunto de actividades o ejercicios preliminares a toda actividad física o deportiva, que pueden ser de carácter general y específicos (Fletcher Monte-Colombo, 2010). El calentamiento 
general está caracterizado por ejercicios de tipo aeróbico de baja o moderada intensidad, mientras que el calentamiento específico puede incluir una combinación de estiramientos dinámicos balísticos y acciones musculares específicas para las demandas del deporte, proporcionando, de esta manera, aumentos agudos en la flexibilidad, la potencia muscular y el rendimiento de salto (Faigenbaum et al., 2006; Silva et al., 2018; Turki et al., 2011). Sin embargo, la magnitud de los efectos del calentamiento puede estar determinada por la selección de los ejercicios, la intensidad, la duración o el tiempo de transición después del calentamiento (McGowan, Pyne, Thompson \& Rattray, 2015).

Actualmente, hay evidencia la cual recomienda que, en deportes que implican gestos dependientes de la velocidad o la fuerza, se debe favorecer la inclusión de ejercicios con acciones musculares específicas a las demandas del deporte, durante el calentamiento específico (Andrade et al., 2015). La inclusión de distintos tipos de ejercicios de fuerza utilizando el peso corporal o cargas externas, con la intensidad y repeticiones específicas, genera un medio óptimo para la producción de energía, al mejorar la función neuromuscular, induciendo a un fenómeno denominado potenciación posactivación (PAP) (Faigenbaum et al., 2006), que lleva a aumentar el rendimiento de salto y sprint (Silva et al., 2018; Till \& Cooke, 2009). El PAP provoca un ascenso agudo en el rendimiento muscular, asociado a una contracción previa. Esto ha sido explicado por distintos mecanismos fisiológicos como un aumento en la fosforilización de las cadenas ligeras que regulan la miosina, incremento del reclutamiento de unidades motoras de orden superior o también cambios en el ángulo de penación (Healy y Comyns, 2017; Hodgson, Docherty y Robbins, 2005; Tillin y Bishop, 2009). En general, se han utilizado distintos mecanismos para generar el PAP, como pesos libres para realizar back squat o power cleans, pliometría con el peso corporal o utilizando chalecos ponderados, o carreras con trineos de arrastre (Healy y Comyns, 2017), por lo que existen diferencias en cuanto a las cargas, cantidad de repeticiones y tiempo de descanso necesario (Gołaś, Maszczyk, Zajac, Mikołajec \& Stastny, 2016; Healy \& Comyns, 2017; Kobal et al., 2019; Seitz \& Haff, 2016).

En los últimos años se ha investigado la utilización de halteras de mano como carga adicional en el entrenamiento con saltos horizontales, la que resulta ser una forma innovadora y eficaz para proporcionar una mejora en el rendimiento deportivo del atleta (Rosas et al., 2016). El uso de halteras de mano ha reportado efectos agudos positivos sobre la mecánica de salto horizontal y, por tanto, en la distancia alcanzada (Cronin, Brughelli, Gamble, Brown \& McKenzie, 2014). Se ha propuesto distintas teorías que explican estos efectos agudos: (a) teoría del tirón, provocado por el balanceo de los brazos; (b) un aumento en el punto de torque, que genera una desaceleración de la contracción muscular, aumentando la fuerza de contracción por la relación fuerza/velocidad; (c) teoría Hold Back, que permite una proyección del centro de masas adecuado para la proyección horizontal del salto, $\mathrm{y}(\mathrm{d})$ teoría del ángulo de despegue óptimo para una mayor proyección (McKenzie, Brughelli, Gamble \& Whatman, 2014). Mackenzie et al, (2016) estudió los efectos de las halteras de mano durante un salto horizontal, sobre la habilidad técnica y las fuerzas de reacción del suelo; encontró que existe un aumento en la producción de fuerza horizontal durante la etapa excéntrica y un incremento en la producción de fuerza horizontal. Cronin et al. (2014) analizó los efectos agudos sobre la cinética y cinemática del salto horizontal con halteras de mano; halló un aumento en la fuerza de reacción del suelo vertical y un acrecentamiento en la fuerza de impulso tanto vertical como horizontal. Estos efectos agudos han llevado a plantear que podrían ayudar a mejorar la capacidad técnica y física, para producir fuerzas horizontales durante la actividad deportiva (Mckenzie et al., 2016). Hay evidencia de que un entrenamiento de saltos cargados con halteras de mano ha mejorado el salto vertical, el salto horizontal y la velocidad en deportistas jóvenes (Rosas et al., 2016); sin embargo, no existen estudios que involucren las halteras de mano para la aplicación de un calentamiento en una sesión o competición. Por lo tanto, este estudio tuvo como objetivo analizar y comparar los efectos de un calentamiento específico con aplicación de saltos cargados con halteras de mano y saltos sin carga sobre el rendimiento en el tiempo de sprint en 20 metros, saltos horizontales y verticales en jugadores de balonmano de la categoría juvenil. Investigaciones previas en torno a los efectos de un calentamiento, que incluye distintos tipos de salto y los efectos agudos de este con utilización de halteras de mano, podrían 
MHSALUD, ISSN: 1659-097X, 17(1), ENERO-JUNIO, 2020, PP 1-12

Gómez-Álvarez, Moyano, Huichaqueo,Veruggio, Urrutia, Hermosilla-Palma, Pavez-Adasme

generar la hipótesis de que un calentamiento con cargas externas es capaz de generar un efecto positivo sobre el rendimiento físico, explicado por el efecto PAP.

\section{Metodología}

\section{Participantes}

El estudio fue un ensayo de tipo experimental con un diseño crossover aleatorizado, todos los sujetos participaron de controles e intervenciones experimentales. Este diseño es utilizado para grupos pequeños de muestra que permiten comparar los resultados de 3 tipos de calentamientos previos y posteriores al calentamiento (Hilfiker, Hübner, Lorenz \& Marti, 2007).

Se reclutaron 13 deportistas de la selección masculina de un equipo de balonmano juvenil (edad: 16,6 \pm 1,33 años, estatura: $169,29 \pm 6,5 \mathrm{~cm}$, masa corporal: $68,99 \pm 10,5 \mathrm{~kg}$ ), actuales campeones nacionales de la disciplina, de los cuales 3 son seleccionados nacionales. El estudio se realizó de acuerdo con la Declaración de Helsinki y todos firmaron un asentimiento informado y un consentimiento informado por parte de los padres o tutores. Antes de las sesiones de prueba, todos los sujetos fueron instruidos para evitar actividad física intensa 48 horas antes de las mediciones y no tomar café o bebidas energéticas al menos 2 horas previas (Andrade et al., 2015).

Todos los participantes cumplieron con criterios de inclusión y exclusión. Criterios de inclusión: (1) ser jugadores activos de la sección de balonmano, (2) no presentar alguna lesión durante los últimos 6 meses (3), contar con el consentimiento y asentimiento informados. Criterios de exclusión: (1) no cumplir con las indicaciones previas a las sesiones de prueba, (2) presentar alguna dolencia o enfermedad aguda en el momento de una intervención.

\section{Procedimientos}

El estudio se ejecutó en el gimnasio donde regularmente entrena el equipo, por lo que se les pidió a los participantes que llegaran media hora antes de su entrenamiento habitual (18:30 horas). Se trabajó con el grupo completo de deportistas durante 4 semanas, haciendo intervenciones de 2 a 3 veces cada semana. 2 intervenciones fueron de familiarización con los saltos utilizados en el estudio, para continuar el estudio con 3 protocolos de calentamientos distintos, realizados durante días no consecutivos, con un periodo de descanso de mínimo 48 horas entre cada sesión (Figura 1).

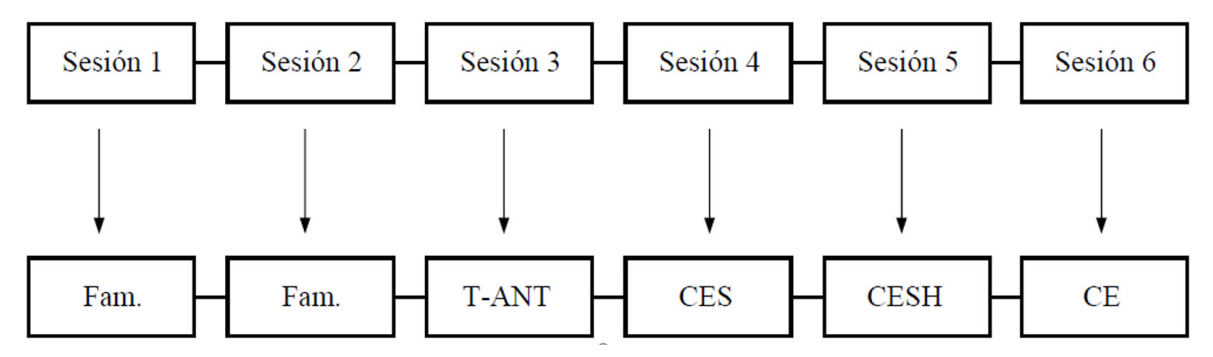

FIGURA 1

Procedimiento por sesión

FAM: familiarización. T-ANT: test antropométricos. CES: calentamiento con estiramientos y saltos. $\mathrm{CESH}$ : calentamiento con estiramientos y saltos con halteras. CE: calentamiento con estiramientos. 
Se utilizó un calentamiento base que incluía correr a una velocidad autoseleccionada, ejercicios de estiramientos, los que fueron previamente descritos (Turki et al., 2012), más otros de saltos horizontales y verticales con o sin carga.

Protocolo 1. Correr y estirar (CE): Primero realizaron un trote de 5 minutos a una velocidad autoseleccionada, para luego dar inicio a estiramiento de tipo dinámico que involucró aducción de piernas ( 5 $\mathrm{x}$ ext.), $40 \mathrm{M}$ de abducción de piernas, $40 \mathrm{M}$ de aducción de piernas, aducciones laterales de piernas ( $5 \mathrm{x}$ ext.), $40 \mathrm{M}$ de talones a los glúteos, $40 \mathrm{M}$ de media tijera, balanceo externo (10 x ext.), $40 \mathrm{M}$ de circunducción de hombros anterior y $40 \mathrm{M}$ de circunducción de hombros posterior.

Protocolo 2. Correr, estirar y saltos sin carga (CES): A los ejercicios incluidos en el protocolo CE se añadieron los siguientes saltos sin carga: horizontales ( 4 series de 5 repeticiones continuas) y verticales ( 4 series de 5 repeticiones continuas). Estos 2 tipos de saltos realizados de forma intercalada, haciendo un total de 80.

Protocolo 3. Correr, estirar y saltos con halteras (CESH): A los ejercicios incluidos en el protocolo CE se añadieron los siguientes saltos cargados con haltera de mano, con un peso adicional de un $8 \%$, con respecto a la masa corporal: horizontales ( 4 series de 5 repeticiones continuas) y verticales ( 4 series de 5 repeticiones continuas). Estos 2 tipos de saltos realizados de forma intercalada, haciendo un total de 80 saltos.

\section{Instrumentos}

Para evaluar el efecto de cada calentamiento, se llevaron a cabo 3 evaluaciones previas y posteriores a una pausa de 3 minutos luego del calentamiento. Estas fueron: salto horizontal, salto vertical y test de velocidad en 20 metros.

Salto de contramovimiento (CMJ): Se utilizó para evaluar el rendimiento de la altura máxima de salto que requiere una acción de ciclo de acortamiento-estiramiento. La prueba CMJ se realizó empleando un sistema de alfombras de contacto electrónico DMJump, usado anteriormente en otras investigaciones (Véliz, Cid, Contreras \& Lagos, 2016; Zapata-Lamana, et al., 2016). Durante el salto CMJ, los participantes recibieron instrucciones de descansar las manos en las caderas, con los pies y los hombros bien separados; realizaron un movimiento hacia abajo, sin ninguna restricción impuesta sobre el ángulo de la rodilla alcanzado, seguido de un salto vertical de máximo esfuerzo (Spurrs, Murphy \& Watsford, 2003). Todos los sujetos fueron instruidos para aterrizar en posición vertical y doblar las rodillas después del aterrizaje con $30 \mathrm{~s}$ de descanso pasivo entre ellos. Se midieron 4 saltos: 2 previos y 2 posteriores al calentamiento.

Salto horizontal (SH): Se aplicó para evaluar la distancia de proyección máxima. El test se realizó con una cinta métrica milimetrada a nivel de suelo, delimitando la punta de los pies en el punto 0 de la huincha (McKenzie et al., 2014), con el fin de iniciar el salto por medio de balanceos de brazos para proyectarlo, cuando los brazos están apuntando hacia el frente, con un ángulo de $45^{\circ} \mathrm{y}$, así, aprovechar el impulso. Todos los sujetos fueron instruidos para aterrizar y marcar, por medio de su talón, la medida de su salto. Con $90 \mathrm{~s}$ de descanso pasivo entre ellos, fueron hechos 4 saltos, 2 precalentamiento y 2 poscalentamiento.

Sprint en 20 metros: Se midió el tiempo empleado en realizar un sprint de 20 metros. En este test, se utilizó un cronometro de mano para la medición (Burgess, Vadachalam, Buchholtz \& Jelsma, 2019; Coleman \& Amonette, 2015; Humberto, Deantonio y Amú-Ruiz, 2016). El inicio fue indicado por un evaluador en el comienzo del recorrido, por medio del sonido del silbato. En el final del recorrido limitado por conos, se encuentra otro evaluador registrando el tiempo, mediante el cronómetro; a medida que se pasa este límite, fueron registrados 2 tiempos (previo y posterior al calentamiento). 


\section{Análisis estadístico}

Los datos se analizaron utilizando el paquete estadístico para ciencias sociales (SPSS) versión 21. Los resultados se expresan como media y desviación estándar. Para evaluar la normalidad de esos datos, se utilizó la prueba de Shapiro-Wilk. La comparación de los resultados entre los 3 protocolos de calentamiento utilizó un ANOVA de 3 vías para medidas repetidas [3 protocolos de calentamiento $\mathrm{x} 2$ veces (es decir, antes y después de los protocolos)]; el nivel alfa se estableció en $\mathrm{p}<0.05$. El análisis del efecto $\mathrm{D}$ de Cohen (TE) también se realizó utilizando el siguiente sistema de clasificación: $\leq 0.19$ (trivial); 0.2-0.59 (pequeño); 0.6-1.19 (moderado); 1.2-1.99 (grande); $\geq 2$ (muy grande) (Hopkins, Marshall, Batterham \& Hanin, 2009).

\section{Resultados}

Para ver los efectos de los calentamientos sobre la capacidad de salto y de la velocidad, se realizaron pruebas previas y posteriores al calentamiento de 13 deportistas. Aquellos incluidos para la investigación presentan una edad promedio de 16,6 $\pm 1,33$ años, un peso promedio de $68,99 \pm 10,59$ kilogramos y la talla media de $169,25 \mathrm{~cm}$. Su porcentaje de masa muscular es de 53,6 $\pm 14,18 \%$ y el de masa magra, de $13 \pm 6,05 \%$ (Tabla 1 ).

TABLA 1

Características demográficas

\begin{tabular}{lc}
\hline Características demográficas & Media (D.E) \\
\hline Edad $(\mathrm{años})$ & $16,6 \pm 1,33$ \\
Peso $(\mathrm{kg})$ & $68,99 \pm 10,5$ \\
Talla $(\mathrm{cm})$ & $169,25 \pm 6,5$ \\
IMC $\left(\mathrm{kg} / \mathrm{m}^{2}\right)$ & $23,9 \pm 2,6$ \\
M.M $(\%)$ & $53,6 \pm 14,18$ \\
M.G $(\%)$ & $13 \pm 6,05$ \\
\hline
\end{tabular}

IMC: índice de masa corporal. M.M: masa muscular. M.G: masa magra.

Al comprar los 3 protocolos de calentamiento (CES, CESH, CE), se puede observar que hubo diferencias significativas en los resultados del SH que muestran cambios significativos en dichos protocolos $(\mathrm{p}<0,05)$ (Tabla 2). Los tamaños del efecto con D de Cohen, con respecto al SH, muestran balances entre los calentamientos, por lo que se obtienen modificaciones moderadas para CES (TE $=-0,85$; diferencia $0.09 \pm$ $0.12)$ y CESH (TE = -0.95; diferencia $0,12 \pm 0,16)$, mientras que el CE obtuvo alteraciones pequeñas (TE $=-0,49$; diferencia $0,07 \pm 0,16$ ) (ver Tabla 2 ).

Los resultados de los protocolos de calentamiento sobre el salto CMJ y la velocidad en sprint muestran diferencias significativas para CES ( $p<0.01$; diferencia CMJ: $2,95 \pm 0.56$ y sprint: $0,29 \pm 0,31$ ) y CESH ( $p$ $<0,01$; diferencia CMJ: 3,50 \pm 0.73 y sprint: $0,20 \pm 0,26)$, no así para $\mathrm{CE}(\mathrm{p}=0,98 ; \mathrm{p}=0,89)$ (tabla 2). En el análisis del tamaño del efecto de la muestra, se obtuvieron valores en la $\mathrm{D}$ de Cohen de carácter moderado, en CMJ para los protocolos CES $(\mathrm{TE}=-0,63)$ y CESH $(\mathrm{TE}=-0,70)$, mientras que el CE obtuvo un efecto trivial $(\mathrm{TE}=-0,00)$. En el tiempo del sprint de 20 metros, se pudo apreciar distinciones de resultados entre protocolos, el CES obtuvo un efecto grande $(\mathrm{TE}=1,28)$, mientras que CESH obtuvo cambios moderados $(\mathrm{TE}=0,78)$ y $\mathrm{CE}$, un efecto pequeño $(\mathrm{TE}=-0,03)$, respectivamente $($ Tabla 2$)$. 
TABLA 2

Comparación de distancia alcanzada en salto horizontal, alturas de salto en CMJ y velocidad antes de calentamiento (PRE-) y después de calentamiento (POS-)

\begin{tabular}{|c|c|c|c|c|c|c|c|c|c|c|c|c|}
\hline & \multicolumn{4}{|c|}{ CES } & \multicolumn{4}{|c|}{ CESH } & \multicolumn{4}{|c|}{ CE } \\
\hline & \multicolumn{4}{|c|}{ Media \pm DE } & \multicolumn{4}{|c|}{ Media $\pm \mathrm{DE}$} & \multicolumn{4}{|c|}{ Media \pm DE } \\
\hline & PRE & POST & DIF & TE & PRE & POST & DIF & TE & PRE & POST & DIF & TE \\
\hline SH & $\begin{array}{c}2,08 \\
\pm \\
0,10\end{array}$ & $\begin{array}{c}2,17 \pm \\
0,11^{*}\end{array}$ & $\begin{array}{c}0,09 \\
\pm \\
0,12\end{array}$ & $\begin{array}{c}- \\
0,8 \\
5\end{array}$ & $\begin{array}{c}2,02 \\
\pm \\
0,12\end{array}$ & $\begin{array}{c}2,15 \pm \\
0,15^{*}\end{array}$ & $\begin{array}{c}0,12 \\
\pm \\
0,16\end{array}$ & $\begin{array}{c}0,9 \\
5\end{array}$ & $\begin{array}{c}2,10 \\
\pm \\
0,14\end{array}$ & $\begin{array}{c}2,17 \pm \\
0,14^{*}\end{array}$ & $\begin{array}{c}0,07 \\
\pm \\
0,16\end{array}$ & $\begin{array}{c}- \\
0,4 \\
9\end{array}$ \\
\hline CMJ & $\begin{array}{c}33,1 \\
6 \pm \\
4,20\end{array}$ & $\begin{array}{c}36,11 \\
\pm \\
5,11^{*}\end{array}$ & $\begin{array}{c}2,95 \\
\pm \\
0,56\end{array}$ & $\begin{array}{c}- \\
0,6 \\
3\end{array}$ & $\begin{array}{c}32,1 \\
7 \pm \\
4,19\end{array}$ & $\begin{array}{c}35,65 \\
\pm \\
5,59^{*}\end{array}$ & $\begin{array}{c}3,50 \\
\pm \\
0,73\end{array}$ & $\begin{array}{c}- \\
0,7 \\
0\end{array}$ & $\begin{array}{c}34,7 \\
0 \pm \\
5,70\end{array}$ & $\begin{array}{c}34,72 \\
\pm 6,21\end{array}$ & $\begin{array}{c}0,19 \\
\pm \\
1,98\end{array}$ & $\begin{array}{c}- \\
0,0 \\
0\end{array}$ \\
\hline VEL & $\begin{array}{c}3,79 \\
\pm \\
0,21\end{array}$ & $\begin{array}{c}3,50 \pm \\
0,24 *\end{array}$ & $\begin{array}{c}0,29 \\
\pm \\
0,31\end{array}$ & $\begin{array}{c}1,2 \\
8\end{array}$ & $\begin{array}{c}3,81 \\
\pm \\
0,26\end{array}$ & $\begin{array}{c}3,61 \pm \\
0,25^{*}\end{array}$ & $\begin{array}{c}0,20 \\
\pm \\
0,26\end{array}$ & $\begin{array}{c}0,7 \\
8\end{array}$ & $\begin{array}{c}3,65 \\
\pm \\
0,28\end{array}$ & $\begin{array}{c}3,66 \pm \\
0,25\end{array}$ & $\begin{array}{c}0,09 \\
\pm \\
0,06\end{array}$ & $\begin{array}{c}- \\
0,0 \\
3\end{array}$ \\
\hline
\end{tabular}

CES: calentamiento estiramiento y saltos. CESH: calentamiento, estiramiento y saltos con halteras de mano. CE: calentamiento y estiramiento. P: ANOVA. TE: tamaño del efecto. ${ }^{*}$ Diferencias significativas $(\mathrm{p}<0,05)$

\section{Discusión}

El propósito de esta investigación fue analizar y comparar los efectos de un calentamiento con aplicación de saltos cargados y sin carga sobre el rendimiento en el sprint de 20 metros, saltos horizontales y verticales, en jugadores de balonmano de la categoría juvenil Dinabec. Los resultados muestran cambios significativos en el protocolo CESH y CES en las evaluaciones de SH, CMJ y tiempo en el sprint de 20 metros, con un TE de moderado a grande.

Los calentamientos que incluyeron ejercicios de salto mostraron ser más eficaces que solo correr y estirar. Previamente, un estudio comparó los efectos de un calentamiento general con uno específico que incluía ejercicios de saltos; encontró un aumento en la altura de saltos CMJ y drop jump, reflejando un aumento en el rendimiento muscular explosivo de las extremidades inferiores (Andrade et al., 2015). Otros trabajos también justifican la aplicación de ejercicios de salto en el calentamiento específico, para mejorar velocidad, salto vertical y agilidad (Andrade et al., 2015; Fletcher \& Monte-Colombo, 2010), los que podrían estar asociados al efecto agudo provocado por el PAP (Faigenbaum et al., 2006) o incluso a una mejora técnica en la producción de impulsos tanto verticales como horizontales, generados por el ejercicio de salto horizontal (Cronin et al., 2014; McKenzie, Brughelli, Whatman y Brown, 2016).

Los resultados muestran que el CES y CESH fueron igual de efectivos sobre las medidas de rendimiento físico evaluadas. Estudios anteriores, que han aplicado saltos con utilización de cargas externas durante el calentamiento, concluyen que la utilización de cargas es más beneficiosa para el deportista (Burkett, Phillips \& Ziuraitis, 2005; Chattong, Brown, Coburn \& Noffal, 2010). Estas investigaciones, por lo demás, obtuvieron, igualmente, resultados positivos para los saltos sin carga; no obstante, el protocolo cargado obtuvo resultados más efectivos; esto podría estar asociado a una mayor producción de fuerza, debido a 
más excitación neuromuscular ligada que impulsará una potenciación posactivación (Burkett et al., 2005; Chattong et al., 2010; Maloney, Turner \& Fletcher, 2014). En relación con esto, protocolos PAP basados en saltos con autocarga y el $10 \%$ del peso corporal fueron efectivos para producir mejoras en la velocidad en 10 y 20 metros (Turner, Bellhouse, Kilduff \& Russell, 2015). En el presente estudio, utilizamos 3 minutos de descanso luego del protocolo, para volver a evaluar a los participantes. Es posible que, si hubiésemos integrado más periodos de evaluación con distintos tiempos de descanso, pudiésemos haber encontrado algunas diferencias significativas. Kilduff et al. (2007) descubrieron que un periodo de descanso igual a 10 minutos, luego del calentamiento, fue el óptimo para identificar los efectos del primero. Aun así, se han reportado incrementos en la altura del salto, evaluada mediante saltos verticales sin contramovimiento, desde los 3 minutos posteriores a la aplicación del protocolo de activación (Seitz, De Villarreal \& Haff, 2014). Sin embargo, los mayores tamaños del efecto se reportan con pausas a partir de los 5 minutos (entre 5 y 7 minutos) (Seitz \& Haff, 2016). Por otro lado, la carga utilizada (8 \% del peso corporal) podría ser reemplazada por otros valores, buscando aquella óptima para cada sujeto, con el fin de optimizar los efectos del calentamiento con utilización de halteras de mano (Cilli, Gelen, Yildiz, Saglam \& Camur, 2014; Kobal, Pereira, Zanetti, Ramírez-Campillo \& Loturco, 2017).

El uso de halteras de mano ha mostrado una mejora en el rendimiento de los saltos, cuando se trata de estímulos agudos (McKenzie et al., 2014). Los resultados positivos van de la mano con la técnica de ejecución del salto: al respetar el protocolo, se demostró que hay una mejora significativa en este salto (McKenzie, Brughelli, Whatman \& Brown, 2015; Randell, Cronin, Keogh, Gill \& Pedersen, 2011). Estos beneficios podrían ser utilizados en los protocolos de calentamiento de distintos deportes.

\section{ConCLusión}

En conclusión, un calentamiento específico que incluye saltos horizontales es eficaz para mejorar SH, CMJ y velocidad en 20 metros. La utilización de halteras de mano, durante la ejecución de saltos a lo largo del calentamiento, no muestra diferencias significativas en los resultados en sprint de $20 \mathrm{~m}$, salto vertical $\mathrm{u}$ horizontal, en comparación con el grupo que incluyó saltos sin carga. Los efectos de la inclusión de saltos en el calentamiento podrían estar asociados a una secuela de PAP.

\section{Recomendaciones}

En el futuro, se sugiere seguir investigando acerca del uso de cargas de halteras de mano en los calentamientos; así también, el peso adecuado para el deportista, ya que ninguna indagación ha podido concluir el peso exacto para maximizar sus efectos positivos (Cilli et al., 2014; Kobal et al., 2017; Rosas et al., 2016). Además, se debería analizar qué tanto influye la experiencia en entrenamiento de saltos o nivel competitivo del deportista sobre los efectos agudos de ejercicios con saltos que utilizan halteras.

\section{REFERENCIAS}

Andrade, D. C., Henriquez-Olguín, C., Beltrán, A. R., Ramírez, M. A., Labarca, C., Cornejo, M., ... Ramírez-Campillo, R. (2015). Effects of general, specific and combined warm-up on explosive muscular performance. Biology of sport, 32(2), 123-128. https://doi.org/10.5604/20831862.1140426

Burgess, T., Vadachalam, T., Buchholtz, K. \& Jelsma, J. (2019). The effect of the contract-relax-antagonist-contract (CRAC) stretch of hamstrings on range of motion, sprint and agility performance in moderately active males: A randomised control trial. South African Journal of Sports Medicine, 31(1), 1-5. https://doi.org/10.17159/20 $78-516 \mathrm{X} / 2019 / \mathrm{v} 31 \mathrm{ila} 6091$ 
MHSALUD, ISSN: 1659-097X, 17(1), ENERO-JUNIO, 2020, PP 1-12 Gómez-Álvarez, Moyano, Huichaqueo,Veruggio, Urrutia, Hermosilla-Palma, Pavez-Adasme

Burkett, L. N., Phillips, W. T., \& Ziuraitis, J. (2005). The Best Warm-Up for the Vertical Jump in College-Age Athletic Men. The Journal of Strength and Conditioning Research, 19(3), 673. https://doi.org/10.1519/15204.1

Chattong, C., Brown, L. E., Coburn, J. W., \& Noffal, G. J. (2010). Effect of a Dynamic Loaded Warm-Up on Vertical Jump Performance. Journal of Strength and Conditioning Research, 24(7), 1751-1754. https://doi.org/10.1519 /JSC.0b013e3181ddf665

Cilli, M., Gelen, E., Yildiz, S., Saglam, T., \& Camur, M. (2014). Acute effects of a resisted dynamic warm-up protocol on jumping performance. Biology of sport, 31(4), 277-282. https://doi.org/10.5604/20831862.1120935

Coleman, A. E., \& Amonette, W. E. (2015). Sprint Accelerations to First Base Among Major League Baseball Players With Different Years of Career Experience. Journal of Strength and Conditioning Research, 29(7), 1759-1765. https://doi.org/10.1519/JSC.0000000000000731

Cronin, J. B., Brughelli, M., Gamble, P., Brown, S. R., \& McKenzie, C. (2014). Acute kinematic and kinetic augmentation in horizontal jump performance using haltere type handheld loading. Journal of Strength and Conditioning Research, 28(6), 1559-1564. https://doi.org/10.1519/JSC.0000000000000312

Faigenbaum, A. D., McFarland, J. E., Schwerdtman, J. A., Ratamess, N. A., Kang, J., \& Hoffman, J. R. (2006). Dynamic warm-up protocols, with and without a weighted vest, and fitness performance in high school female athletes. Journal of athletic training, 41(4), 357-363. Recuperado de http://www.ncbi.nlm.nih.gov/pubmed/17273458

Fletcher, I. M., \& Monte-Colombo, M. M. (2010). An Investigation into the Effects of Different Warm-Up Modalities on Specific Motor Skills Related to Soccer Performance. Journal of Strength and Conditioning Research, 24(8), 2096-2101. https://doi.org/10.1519/JSC.0b013e3181e312db

Gołaś, A., Maszczyk, A., Zajac, A., Mikołajec, K., \& Stastny, P. (2016). Optimizing post activation potentiation for explosive activities in competitive sports. Journal of Human Kinetics, 52(1), 95-106. https://doi.org/10.1515/ hukin-2015-0197

Healy, R. \& Comyns, T. M. (2017). The application of postactivation potentiation methods to improve sprint speed. Strength and Conditioning Journal, 39(1), 1-9. https://doi.org/10.1519/SSC.0000000000000276

Hilfiker, R., Hübner, K., Lorenz, T. \& Marti, B. (2007). Effects of Drop Jumps Added to the Warm-Up of Elite Sport Athletes With a High Capacity for Explosive Force Development. The Journal of Strength and Conditioning Research, 21(2), 550. https://doi.org/10.1519/R-20215.1

Hodgson, M., Docherty, D. \& Robbins, D. (2005). Post-activation potentiation: Underlying physiology and implications for motor performance. Sports Medicine 35, 585-595. https://doi.org/10.2165/00007256-20053 5070-00004

Hopkins, W., Marshall, S., Batterham, A. \& Hanin, J. (2009). Progressive Statistics for Studies in Sports Medicine and Exercise Science. Medicine \& Science in Sports \& Exercise, 41(1), 3-13. https://doi.org/10.1249/MSS.0b0 $13 \mathrm{e} 31818 \mathrm{cb} 278$

Humberto, J., Deantonio, L. y Amú-Ruiz, F. A. (2016). Características morfofuncionales y motoras de los seleccionados deportivos de la Universidad del Valle. Revista Cientifica General José María Córdova, 14, 169-193.

Kilduff, L. P., Bevan, H. R., Kingsley, M. I. C., Owen, N. J., Bennett, M. A., Bunce, P. J., ... Cunningham, D. J. (2007). Postactivation Potentiation in Professional Rugby Players: Optimal Recovery. The Journal of Strength and Conditioning Research, 21(4), 1134. https://doi.org/10.1519/R-20996.1

Kobal, R., Pereira, L. A., Kitamura, K., Paulo, A. C., Ramos, H. A., Carmo, E. C., ... Loturco, I. (2019). Post-Activation Potentiation: Is there an Optimal Training Volume and Intensity to Induce Improvements in Vertical Jump Ability in Highly-Trained Subjects? Journal of Human Kinetics, 66(1), 195-203. https://doi.org/10.2478/huk in-2018-0071

Kobal, R., Pereira, L. A., Zanetti, V., Ramírez-Campillo, R. \& Loturco, I. (2017). Effects of Unloaded vs. Loaded Plyometrics on Speed and Power Performance of Elite Young Soccer Players. Frontiers in Physiology, 8, 742. h ttps://doi.org/10.3389/fphys.2017.00742

Maloney, S. J., Turner, A. N. \& Fletcher, I. M. (2014). Ballistic Exercise as a Pre-Activation Stimulus: A Review of the Literature and Practical Applications. Sports Medicine, 44(10), 1347-1359. https://doi.org/10.1007/s4027 9-014-0214-6 
MHSALUd, ISSN: 1659-097X, 17(1), ENERo-Junio, 2020, PP 1-12

Gómez-Álvarez, Moyano, Huichaqueo,Veruggio, Urrutia, Hermosilla-Palma, Pavez-Adasme

McGowan, C. J., Pyne, D. B., Thompson, K. G. y Rattray, B. (2015). Warm-Up Strategies for Sport and Exercise: Mechanisms and Applications. Sports Medicine, 45(11), 1523-1546. https://doi.org/10.1007/s40279-015-037 6-x

McKenzie, C., Brughelli, M., Gamble, P. \& Whatman, C. (2014). Enhancing Jump Performance With Handheld Loading. Strength and Conditioning Journal, 36(2), 32-38. https://doi.org/10.1519/SSC.0000000000000041

McKenzie, C., Brughelli, M., Whatman, C. \& Brown, S. (2015). The Influence of Optimal Handheld Load on the Technical Ability to Apply Ground Reaction Forces during Horizontal Jumping in Female Netball Players. International Journal of Sports Medicine, 37(04), 318-323. https://doi.org/10.1055/s-0035-1565052

McKenzie, C., Brughelli, M., Whatman, C. \& Brown, S. R. (2016). The Influence of Optimal Handheld Load on the Technical Ability to Apply Ground Reaction Forces during Horizontal Jumping in Female Netball Players. International Journal of Sports Medicine, 37(4), 318-323. https://doi.org/10.1055/s-0035-1565052

Pagaduan, J. C., Pojskić, H., Užičanin, E. \& Babajić, F. (2012). Effect of Various Warm-Up Protocols on Jump Performance in College Football Players. Journal of Human Kinetics, 35(1), 127-132. https://doi.org/10.2478 /v10078-012-0086-5

Randell, A. D., Cronin, J. B., Keogh, J. W. L., Gill, N. D. \& Pedersen, M. C. (2011). Effect of Instantaneous Performance Feedback During 6 Weeks of Velocity-Based Resistance Training on Sport-Specific Performance Tests. Journal of Strength and Conditioning Research, 25(1), 87-93. https://doi.org/10.1519/JSC.0b013e3181 fee634

Rosas, F., Ramírez-Campillo, R., Díaz, D., Abad-Colil, F., Martínez-Salazar, C., Caniuqueo, A., ... Izquierdo, M. (2016). Jump Training in Youth Soccer Players: Effects of Haltere Type Handheld Loading. International Journal of Sports Medicine, 37(13), 1060-1065. https://doi.org/10.1055/s-0042-111046

Seitz, L. B., De Villarreal, E. S. \& Haff, G. G. (2014). The Temporal Profile of Postactivation Potentiation Is Related to Strength Level. Journal of Strength and Conditioning Research, 28(3), 706-715. https://doi.org/10.1519/JS C.0b013e3182a73ea3

Seitz, L. B. \& Haff, G. G. (2016). Factors Modulating Post-Activation Potentiation of Jump, Sprint, Throw, and Upper-Body Ballistic Performances: A Systematic Review with Meta-Analysis. Sports Medicine, 46(2), 231-240. https://doi.org/10.1007/s40279-015-0415-7

Silva, L. M., Neiva, H. P., Marques, M. C., Izquierdo, M. \& Marinho, D. A. (2018). Effects of Warm-Up, Post-WarmUp, and Re-Warm-Up Strategies on Explosive Efforts in Team Sports: A Systematic Review. Sports Medicine, 48(10), 2285-2299. https://doi.org/10.1007/s40279-018-0958-5

Spurrs, R. W., Murphy, A. J. \& Watsford, M. L. (2003). The effect of plyometric training on distance running performance. European Journal of Applied Physiology, 89(1), 1-7. https://doi.org/10.1007/s00421-002-0741-y

Till, K. A. \& Cooke, C. (2009). The Effects of Postactivation Potentiation on Sprint and Jump Performance of Male Academy Soccer Players. Journal of Strength and Conditioning Research, 23(7), 1960-1967. https://doi.org/10 .1519/JSC.0b013e3181b8666e

Tillin, N. A. \& Bishop, D. (2009). Factors modulating post-activation potentiation and its effect on performance of subsequent explosive activities. Sports Medicine 39(2): 147-166. https://doi.org/10.2165/00007256-2009390 20-00004

Turki, O., Chaouachi, A., Behm, D. G., Chtara, H., Chtara, M., Bishop, D., ... Amri, M. (2012). The Effect of WarmUps Incorporating Different Volumes of Dynamic Stretching on 10- and 20-m Sprint Performance in Highly Trained Male Athletes. Journal of Strength and Conditioning Research, 26(1), 63-72. https://doi.org/10.1519/ JSC.0b013e31821ef846

Turki, O., Chaouachi, A., Drinkwater, E. J., Chtara, M., Chamari, K., Amri, M. \& Behm, D. G. (2011). Ten Minutes of Dynamic Stretching Is Sufficient to Potentiate Vertical Jump Performance Characteristics. Journal of Strength and Conditioning Research, 25(9), 2453-2463. https://doi.org/10.1519/JSC.0b013e31822a5a79

Turner, A. P., Bellhouse, S., Kilduff, L. P. \& Russell, M. (2015). Postactivation Potentiation of Sprint Acceleration Performance Using Plyometric Exercise. Journal of Strength and Conditioning Research, 29(2), 343-350. https: //doi.org/10.1519/JSC.0000000000000647 
MHSALUD, ISSN: 1659-097X, 17(1), ENERo-Junio, 2020, PP 1-12 Gómez-Álvarez, Moyano, Huichaqueo,Veruggio, Urrutia, Hermosilla-Palma, Pavez-Adasme

Véliz, C. V., Cid, F. M., Contreras, V. T. y Lagos, S. F. (2016). Diferencias en saltos verticales continuos durante 15 segundos entre gimnastas, voleibolistas, nadadoras y nadadoras sincronizadas del Estadio Mayor de Santiago de Chile. Ciencias de la Actividad Fisica, 17(1), 49-57. Recuperado de http://revistacaf.ucm.cl/article/view/90

Wagner, H., Finkenzeller, T., Würth, S. \& Von Duvillard, S. P. (2014). Individual and team performance in teamhandball: a review. Journal of sports science \& medicine, 13(4), 808-816. Recuperado de http://www.ncbi.nlm. nih.gov/pubmed/25435773

Zapata-Lamana, R., Monsalves-Álvarez, M., Cigarroa-Cuevas, I., Castro-Sepúlveda, M., Salazar-Rodríguez, G. y Abad-Colil, F. (2016). Diferencias de composición corporal y condición física en educadoras de párvulo con normopeso, sobrepeso y obesidad: Estudio transversal. Revista chilena de nutrición, 43(4), 5-5. https://doi.org/ $10.4067 /$ S0717-75182016000400005

\section{BY-NC-ND}

